\title{
Micromagnetic modeling of laser-induced magnetization dynamics using the Landau-Lifshitz-Bloch equation
}

\author{
U. Atxitia and O. Chubykalo-Fesenko ${ }^{\text {a) }}$ \\ Instituto de Ciencia de Materiales de Madrid, CSIC, Cantoblanco, 28049 Madrid, Spain
}

N. Kazantseva, D. Hinzke, U. Nowak, and R. W. Chantrell

Department of Physics, University of York, York YO10 5DD, United Kingdom

(Received 5 September 2007; accepted 18 November 2007; published online 7 December 2007)

\begin{abstract}
We present a dynamic approach to micromagnetics based on the Landau-Lifshitz-Bloch equation and Langevin dynamics. This type of modeling will be necessary at high temperatures when the magnetization length is not conserved, especially close to the Curie temperature. We model the laser-induced magnetization dynamics with various laser pulse fluences and show that the results are consistent with both experiments and atomistic modeling. Our results show different recovery rates depending on the final demagnetized state. (C) 2007 American Institute of Physics.
\end{abstract}

[DOI: $10.1063 / 1.2822807$ ]

Recent advances in ultrafast pulsed laser experiments in magnetic media have opened new possibilities of controlling magnetization dynamics in the femto- and picosecond regime. ${ }^{1-3}$ An essential part of these experiments is a heat pulse, produced by the laser, acting on the magnetic material. The physical processes governing the magnetization dynamics are complicated and involve photon, electron, phonon, and spin interactions. Laser-induced precession has been observed with low laser pump fluency, and extraordinarily fast all-optical demagnetization has been demonstrated in the femtosecond regime using high pump fluency. During these processes three timescales can be distinguished: an ultrarapid magnetization decay in the femtosecond regime is followed by magnetization recovery on the order of picoseconds. Finally, magnetization precession takes place on the nanosecond timescale as the magnetization moves into equilibrium with the local field.

Although the underlying processes are complicated, magnetization dynamics at high temperatures can reasonably be understood taking into account that during ultrafast laserinduced processes, the electron temperature rapidly increases up to and often above the Curie temperature. On this basis, classical spin models have demonstrated their potential to reproduce the main features of the pump-probe experiment. ${ }^{4}$ However, due to their atomistic nature, the total simulated size in these models constitutes several thousands of cubic nanometers at most. Larger simulation scale is the province of micromagnetics. In this approach, the discretization unit of constant magnetization length represents an average magnetization over the smaller (atomistic) scale. Although the temperature fluctuations can be included via the Langevin approach based on the Landau-Lifshitz-Gilbert (LLG) equation, micromagnetic simulations are not suitable for high temperatures. This is due to the fact that micromagnetic simulations do not include the high-frequency spin waves and, thus, the Curie temperature is seriously overestimated. ${ }^{5}$ Moreover, in recent atomistic simulations, ${ }^{6}$ it has been demonstrated that at high temperatures several important effects occur, which cannot be taken into account in the LLG approach. Namely, during the magnetization dynamics, (i) the magnetization vector magnitude is not conserved, (ii) longitudinal magnetization relaxation occurs with the longitudinal relaxation time increase approaching the Curie temperature (critical slowing down), and (iii) at the same time the transverse relaxation time decreases. It has been shown that all these effects are in agreement with single macrospin dynamics based on the Landau-Lifshitz-Bloch (LLB) equation. This equation was derived by Garanin for classical ${ }^{7}$ and quantum ${ }^{8}$ average spin polarizations. At low temperatures it coincides with the standard LLG equation but it is valid up to and beyond the Curie temperature $T_{C}$. The necessity of the longitudinal relaxation to model the pump-probe experiment via the micromagnetic approach has been noted by Vomin et al. ${ }^{9}$ who suggested to use for this purpose the Bloch equation. The advantage of the LLB equation resides in the fact that it is a much more consistent approach which has a more rigorous foundation and has been tested against the predictions of atomistic modeling. ${ }^{6}$

The purpose of the present letter is to introduce a full micromagnetic approach based on the LLB equation and Langevin dynamics and to show its suitability to model high temperature magnetization dynamics below and above the Curie temperature. As an example, we model experiments on ultrafast laser-induced magnetization precession. To distinguish the approach from normal (LLG-based) micromagnetics, we refer to this as LLB-micromagnetics, or LLB$\mu \mathrm{MAG}$, for convenience in the following.

Our model uses cubic discretization elements with lateral size $\Delta$. For each cube, we write the stochastic LLB equation, describing its average spin polarization $\mathbf{m}_{i}$, in the following form: ${ }^{10}$

$$
\begin{aligned}
\dot{\mathbf{m}}_{i}= & \gamma\left(\mathbf{m}_{i} \times \mathbf{H}_{\mathrm{eff}}^{i}\right)+\frac{\gamma \alpha_{\|}}{m_{i}^{2}}\left[\mathbf{m}_{i} \cdot\left(\mathbf{H}_{\mathrm{eff}}^{i}+\zeta_{\|}^{i}\right)\right] \mathbf{m}_{i}-\frac{\gamma \alpha_{\perp}}{m_{i}^{2}}\left\{\mathbf{m}_{i}\right. \\
& \left.\times\left[\mathbf{m}_{i} \times\left(\mathbf{H}_{\mathrm{eff}}^{i}+\zeta_{\perp}^{i}\right)\right]\right\},
\end{aligned}
$$

where $\gamma$ is the gyromagnetic ratio, $\alpha_{\|}$and $\alpha_{\perp}$ are dimensionless longitudinal and transverse damping parameters given by

$$
\alpha_{\|}=\lambda \frac{2 T}{3 T_{C}},
$$

${ }^{a)}$ Electronic mail: oksana@icmm.csic.es. 


$$
\alpha_{\perp}=\lambda\left(1-\frac{T}{3 T_{C}}\right), \quad T<T_{C},
$$

and $T_{C}$ is the Curie temperature. For $T>T_{C}, \alpha_{\perp}$ equals $\alpha_{\|}$. Here, $\lambda$ is the parameter describing the coupling of the spins to the heat bath at atomistic level. The effective field $\mathbf{H}_{\text {eff }}$ is given by

$$
\begin{aligned}
\mathbf{H}_{\mathrm{eff}}^{i}= & \mathbf{H}+\mathbf{H}_{A}^{i}+\mathbf{H}_{\mathrm{ex}}^{i} \\
& +\left\{\begin{array}{cc}
\frac{1}{2 \tilde{\chi}_{\|}}\left(1-\frac{m_{i}^{2}}{m_{e}^{2}}\right) \mathbf{m}_{i}, & T \lesssim T_{C} \\
\frac{J_{0}}{\mu}\left(1-\frac{T}{T_{C}}-\frac{3}{5} m_{i}^{2}\right) \mathbf{m}_{i}, & T \gtrsim T_{C} .
\end{array}\right.
\end{aligned}
$$

Here, $m_{e}$ is the zero-field equilibrium spin polarization for a given temperature, $J_{0}=z J$ for an atomistic nearest neighbor exchange of Heisenberg type with exchange parameter $J$ and $z$ nearest neighbors, and $\mu$ is the atomistic magnetic moment. $\mathbf{H}, \mathbf{H}_{A}^{i}$, and $\mathbf{H}_{\mathrm{ex}}^{i}$ are applied, anisotropy (in this case the easy axis is parallel to the $z$ direction) and exchange fields, respectively. We suppose the following expressions from meanfield approximation (MFA),

$$
\begin{aligned}
& \mathbf{H}_{A}^{i}=-\left(m_{x}^{i} \mathbf{e}_{x}+m_{y}^{i} \mathbf{e}_{y}\right) / \tilde{\chi}_{\perp}, \\
& \mathbf{H}_{\mathrm{ex}}^{i}=-\frac{A(T)}{m_{e}^{2}} \frac{2}{M_{s}^{0} \Delta^{2}} \sum_{j \in \operatorname{neigh}(i)}\left(\mathbf{m}_{j}-\mathbf{m}_{i}\right),
\end{aligned}
$$

where $\tilde{\chi}_{\|}$and $\tilde{\chi}_{\perp}$ are parallel and perpendicular susceptibilities, $M_{s}^{0}$ is the saturation magnetization value at $T=0$, and $A(T)$ is the micromagnetic exchange. Stochastic fields $\zeta_{\|}$and $\zeta_{\perp}$ describe the dispersion of different magnetization trajectories and have the following properties: ${ }^{10}$

$$
\left\langle\zeta_{a}^{i}(0) \zeta_{a}^{j}(t)\right\rangle=\frac{2 k_{B} T}{\gamma \alpha_{a} M_{s}^{0} V_{i}} \delta_{i j} \delta(t),
$$

where $a$ stands for one of the symbols " "l" or " $\perp$," $i$ and $j$ denote different components $x, y$, and $z$ or different discretization elements, and $V_{i}$ is the discretization element volume. We have also checked that, similar to the LLG case, ${ }^{11}$ the stochastic fields introduced via Eq. (1) do not produce correlations between different magnetization sites.

The integration of the LLB equation requires several temperature dependent parameters. In principle, they can be considered as an input from experimental values or estimated from atomistic modeling. In the present paper, we take these parameters from MFA. Namely, the equilibrium magnetization $m_{e}$ is taken from the Curie-Weiss law $m_{e}=B\left[\left(m_{e} J\right.\right.$ $\left.+\mu H) / k_{B} T\right]$, where $B$ is the Langevin function and $H \rightarrow 0$, and the longitudinal susceptibility as its derivative $\tilde{\chi}_{\|}$ $=\partial m_{e} / \partial H$. For the anisotropy parameter, we assume the approximate relation $K(T) \sim m_{e}^{3}$, ${ }^{12}$ and for the micromagnetic exchange parameter $A(T) \sim m_{e}^{2}$. Note that with these choices we assume the anisotropy and the exchange fields to be zero above $T_{C}$, leaving a more detailed investigation of their properties for future work. Most of the simulations have been performed for $\mathrm{Ni}$ alloys with uniaxial anisotropy $[K(T=0)$ $\left.=5.3 \times 10^{4} \mathrm{erg} / \mathrm{cm}^{3}\right), \quad M_{s}^{0}=480 \mathrm{emu} / \mathrm{cm}^{3}, \quad T_{C}=630 \mathrm{~K}$ $\left(k_{B} T_{C}=2 \mathrm{~J}\right.$ in the MFA), and $\lambda=0.1$.

To check thermal equilibrium properties in a multi macrospin approach, we present in Fig. 1 the thermal average of the magnetization value, obtained by integrating the stochasDownloaded 23 Feb 2010 to 161.111 .180 .191 . Redistribution subject

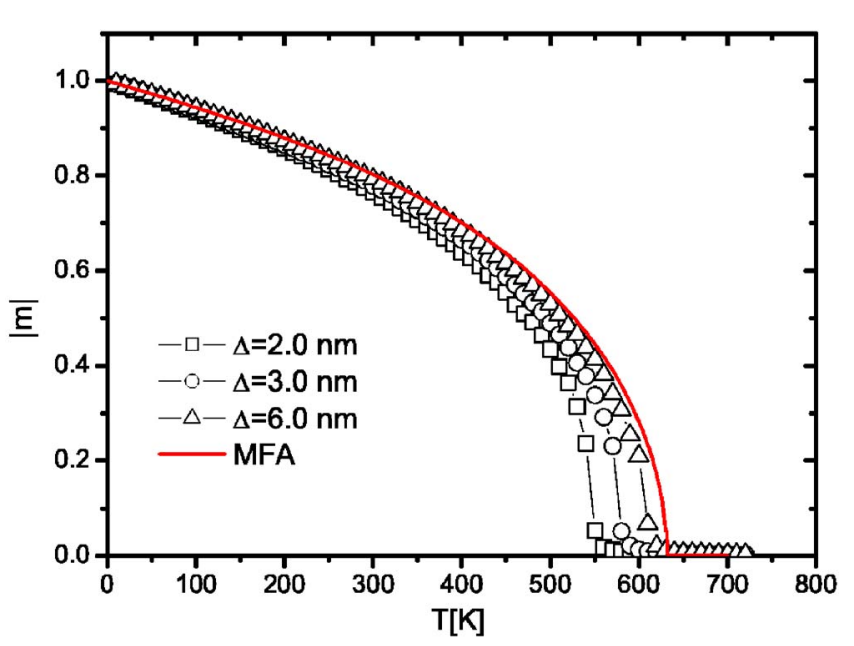

FIG. 1. (Color online) Equilibrium magnetization calculated by means of LLB- $\mu$ MAG in a cube with $24 \mathrm{~nm}$ sides discretized in cubes with different cell sizes $\Delta$. The solid line represents the magnetization in MFA.

tic LLB equations [Eq. (1)] for different cell sizes $\Delta$. As can be seen, the LLB- $\mu$ MAG approach gives reasonably good agreement with the expected behavior, though in the critical region cell-size dependent deviations can be seen. These deviations are due to the fact that the LLB equation was derived within the MFA, hence, strictly speaking, for an infinite system. Consequently, cell sizes have to be large enough so that each single cell behaves like a thermodynamic system. Therefore, the cell size must be larger than the thermal correlation length $\xi(T)$. Since $\xi$ diverges when approaching the Curie temperature finite-size effects lead to a deviation from true critical behavior. For $\Delta$ too small, approaching the limit of atomic spins, the LLB equation can no longer be valid since in this limit the magnitude of the spin moment would be preserved. Hence, in this limit, the magnetic order is underestimated by the LLB approach. Further differences may be attributed to the fact that the micromagnetic exchange $A(T)$ is not known at higher temperatures and some preliminary calculations suggest that it may not be described by a single scaling exponent. Nevertheless, we have found the agreement satisfactory and within the errors introduced by the estimations for other parameters.

Next, we use the LLB- $\mu$ MAG model to study the heatinduced magnetization dynamics produced by laser pulses where we assume Gaussian shape of $50 \mathrm{fs}$ duration and different fluences. During the laser-induced demagnetization, the external temperature is transferred into several subsystems. Similar to Ref. 4, we assume a simple channel where the photon energy is transfered to the electrons and the magnetization is directly coupled to the electron temperature $T_{e}$ which we calculate within the two-temperature model,

$$
\begin{aligned}
& C_{e} \frac{d T_{e}}{d t}=-G_{e l}\left(T_{e}-T_{l}\right)+P(t), \\
& C_{l} \frac{d T_{l}}{d t}=G_{e l}\left(T_{e}-T_{l}\right),
\end{aligned}
$$

where $C_{e}$ and $C_{l}$ are the electron and lattice specific heat constants, $G_{e l}$ is a coupling constant, and $P(t)$ is the laser fluency.

First we model a demagnetization process produced by a laser pulse with high fluency. The results of our simulations to AIP license or copyright; see http://apl.aip.org/apl/copyright.jsp 


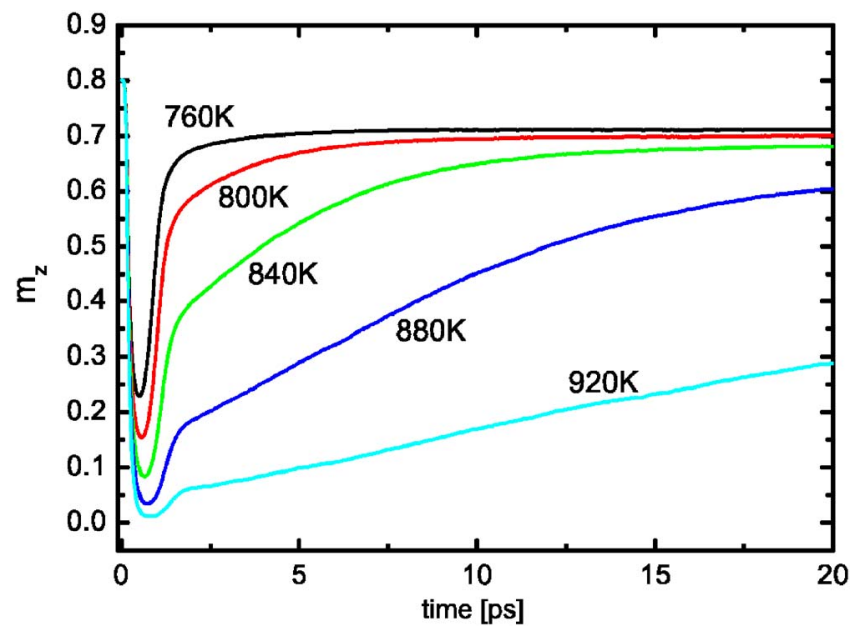

FIG. 2. (Color online) Modeling of laser-induced magnetization dynamics in the same system as in Fig. 1 for different laser fluences. The numbers indicate maximum electron temperatures obtained through the twotemperature model [Eq. (7)].

are presented in Fig. 2. We note here that laser pulses, producing maximum electron temperatures far above the Curie temperature, are required for demagnetization. This is consistent with atomistic simulations, ${ }^{4}$ and is because the rate of energy transfer from the conduction electrons takes place with a characteristic relaxation time determined by the coupling between the conduction electrons and the spins. If the relaxation time $\left(\sim T^{-1}\right)$ is less than the laser pulse width demagnetization will not occur. Essentially the spin temperature lags the electron temperature. This is an important effect which is well-described by the LLB- $\mu$ MAG approach. Our model reproduces all essential features of the heat-assisted demagnetization. Namely, we observe a very fast demagnetization, occurring at femtosecond scale, followed by a more slow (picosecond) recovery. The rate of the recovery is different depending on the minimum magnetization value achieved during the demagnetization. A similar effect has been observed in atomistic simulations. ${ }^{4}$ The observed slow recovery rate is due to the loss of correlations at high temperature since the micromagnetic exchange vanishes. Consequently, additional time is necessary to recover the correlations and, therefore, the magnetization.

In our second calculation, we reproduce the optical ferromagnetic resonance (FMR) experiments reported in Ref. 1. Namely, we assume a thin film and a laser pulse which produces a maximum electron temperature $T_{e}=800 \mathrm{~K}$. For simplicity, the magnetostatic interactions were included assuming an additional easy plane anisotropy $H_{A}=4 \pi M_{s}^{0} m_{e}\left(m_{x} \mathbf{e}_{x}\right.$ $\left.+m_{y} \mathbf{e}_{y}\right)$. An external field of $0.3 \mathrm{~T}$ was applied perpendicular to the plane. Initially at $T=300 \mathrm{~K}$, the equilibrium magnetization direction made an angle of $25^{\circ}$ with respect to the thin film plane. During the laser pulse, the magnetization value rapidly decreases. Consequently, the anisotropy value decreases, the system is no longer in equilibrium and a laser-

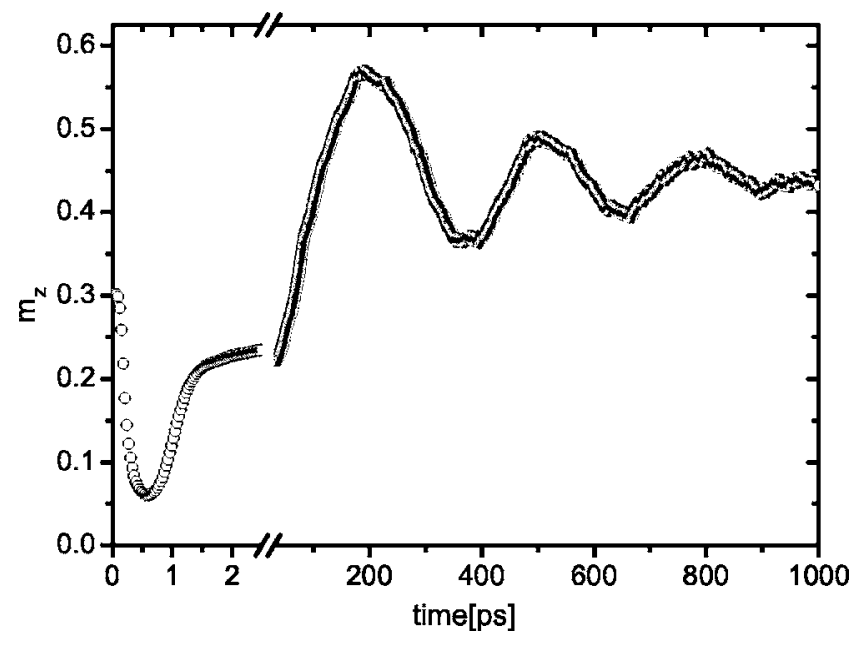

FIG. 3. Modeling of a fast demagnetization dynamics (Ref. 1) with low laser fluency in a thin film $\left(96 \times 96 \times 12 \mathrm{~nm}^{3}\right)$ with periodic boundary conditions and in-plane anisotropy of value $K(T=0)=2.3 \times 10^{5} \mathrm{erg} / \mathrm{cm}^{3}$.

induced precession appears as the magnetization recovers. The results of our simulations are presented in Fig. 3 and are similar to the ones reported experimentally in Ref. 1. We have also found that the precessional frequency decreases with the maximum electron temperature.

In conclusion, we have introduced a micromagnetic approach valid for all temperatures, both below and above the Curie temperature. This approach overcomes the limitations of the standard micromagnetics which is not capable of modeling high temperature magnetization dynamics. Our calculations show that the results of this approach are consistent with both atomistic modeling ${ }^{4}$ and experimental findings. ${ }^{1}$ This opens new possibilities for large-scale simulations at high temperatures.

This work was supported by Seagate Technology. The support of the Royal Society travel grant is also gratefully acknowledged.

${ }^{1}$ M. van Kampen, C. Jozsa, J. T. Kohlhepp, P. LeClair, L. Lagae, W. J. M. de Jonge, and B. Koopmans, Phys. Rev. Lett. 88, 227201 (2002).

${ }^{2}$ E. Beaurepaire, J.-C. Merle, A. Daunois, and J. Y. Bigot, Phys. Rev. Lett. 76, 4250 (1996).

${ }^{3}$ B. Koopmans, M. van Kampen, J. T. Kohlhepp, and W. J. M. de Jonge, Phys. Rev. Lett. 85, 955 (2000).

${ }^{4}$ N. Kazantseva, U. Nowak, R. W. Chantrell, J. Hohlfeld, and A. Rebei, Europhys. Lett. (to be published).

${ }^{5}$ G. Grinstein and R. H. Koch, Phys. Rev. Lett. 90, 207201 (2003).

${ }^{6}$ O. Chubykalo-Fesenko, U. Nowak, R. W. Chantrell, and D. Garanin, Phys. Rev. B 74, 094436 (2006).

${ }^{7}$ D. A. Garanin, Phys. Rev. B 55, 3050 (1997).

${ }^{8}$ D. A. Garanin, Physica A 172, 470 (1991).

${ }^{9}$ M. Vomir, L. H. F. Andrade, L. Guidoni, E. Beaurepaire, and J.-Y. Bigot, Phys. Rev. Lett. 94, 237601 (2005).

${ }^{10}$ D. A. Garanin and O. Chubykalo-Fesenko, Phys. Rev. B 70, 212409 (2004).

${ }^{11}$ O. A. Chubykalo, R. Smirnov-Rueda, J. M. González, M. A. Wongsam, R. W. Chantrell, and U. Nowak, J. Magn. Magn. Mater. 226, 28 (2003).

${ }^{12}$ E. R. Callen and H. B. Callen, J. Phys. Chem. Solids 27, 1271 (1966). 